\title{
Impact of Steps, Instruction, and Motivation on Learning Symbolic Reasoning Using an Online Tool
}

\author{
Megan Fowler, Michelle Cook, Kevin Plis, \\ Tim Schwab, Yu-Shan Sun, Murali Sitaraman \\ School of Computing \\ Clemson University, Clemson, SC USA \\ mefowle@clemson.edu
}

\author{
Jason O. Hallstrom \\ Engineering \& Computer Science \\ FAU, \\ Boca Raton, FL USA \\ jhallstrom@fau.edu
}

\author{
Joseph E. Hollingsworth \\ Computer Science \\ IU Southeast, \\ New Albany, IN USA \\ jholly@ius.edu
}

\begin{abstract}
Several research studies have shown the benefits of code tracing to promote student understanding of program behavior. While code tracing on specific input values is a useful starting point, students ultimately need to be able to reason rigorously and logically about the correctness of their code on all (i.e., arbitrary) inputs. Otherwise, they may make false generalizations and may achieve only a shallow understanding. Results of a multi-semester experiment to answer the following research questions: (1) With or without steps, can students learn the basics of tracing code on symbolic input values using an online tool? And how important is classroom instruction? (2) What is the impact of motivation on student attitudes in learning to reason with such a tool? Data was obtained from 297 subjects who used the online reasoning tool in a second-year software development course for CS majors. Analysis indicates that students can do symbolic reasoning to trace code and that instruction and motivation have significant impact.
\end{abstract}

\section{CCS CONCEPTS}

- Applied computing Computer-assisted instruction

- Theory of computation $\sim$ Program reasoning

\section{KEYWORDS}

Attitudes, correctness, symbolic reasoning, motivation, online tool

ACM Reference Format: M. Fowler, et al. 2018. Impact of Steps, Instruction, and Motivation on Learning Symbolic Reasoning Using an Online Tool. In Proceedings of $50^{\text {th }}$ ACM Technical Symposium on Computer Science Education (SIGCSE '19), February 27-March 2, 2019, Minneapolis, MN, USA. ACM, New York, NY, USA, 7 pages. DOI:

https://doi.org/10.1145/3287324.3287401

Permission to make digital or hard copies of all or part of this work for personal or classroom use is granted without fee provided that copies are not made or distributed for profit or commercial advantage and that copies bear this notice and the full citation on the first page. Copyrights for components of this work owned by others than ACM must be honored. Abstracting with credit is permitted. To copy otherwise, or republish, to post on servers or to redistribute to lists, requires prior specific permission and/or a fee. Request permissions from Permissions@acm.org. SIGCSE '19, February 27-March 2, 2019, Minneapolis, MN, USA

(c) 2019 Association for Computing Machinery.

ACM ISBN 978-1-4503-5890-3/19/02 $\square \$ 15.00$

https://doi.org/10.1145/3287324.3287401

\section{Introduction}

To learn to develop correct software, students need a firm foundation in precise analytical reasoning skills. Analytical reasoning is the bedrock of a good computer science education, but it is difficult to learn. Running programs on select inputs and studying the outputs gives students only limited understanding, yet more thorough symbolic reasoning using paper-and-pencil methods relies on labor-intensive manual checking. The objective of this research is to understand whether we can effectively teach a vast majority of computer science students the basics of reasoning about code on all input values using a symbolic approach with the (non-exclusive) aid of a reasoning tool. The tool has already been shown to be effective in pinpointing specific difficulties for individual students and subgroups [[9]].

This research is motivated in part by the documented benefits of learning to trace code and reason [[5], [17], [20], [24]]. For example, whereas the tool discussed in [[18], [30]] lets students visualize values of variables in their code after each step, the findings in [[44]] note the benefits for learners even within a limited context without the aid of any tools. Consider the following piece of code, where $i$ and $j$ are two integers.

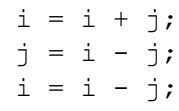

Testing relies on running the code on specific inputs for $i$ and $j$, such as 3 and 5 , and checking the answers, while tracing relies on analyzing the values of $i$ and $j$ after each step on these inputs. A symbolic approach aims for students to learn to generalize the reasoning and understand the general purpose of the code. Here, the goal is for students to learn to reason about the effect of the code supposing the initial values of $i$ and $j$, respectively are some abstract inputs, \# $i$ and $\# j$, and to determine the values of $i$ and $j$ at the end of the code in terms of the abstract input values.

Beyond our own collective experiences in teaching students over multiple decades at diverse institutions, both in person and using online systems, two observations motivate the central objectives and approach of the research project underlying this paper. The first of these is a study of novice programmers at Yale [[39]], where Spohrer and Soloway note the difficulties students face in reasoning about compositions of statements, though they seem to understand the individual programming constructs. At public institutions like ours, such problems are apparent even in 
later CS courses, in spite of our efforts to employ best practices. The second one is a debate in learning theory about the depth of understanding students achieve after abstract versus concrete introductions [[6], [14], [22], [27]], though the setting there is not computing. The findings in [[22]] suggest that "...giving college students multiple concrete examples may not be the most efficient means of promoting transfer of knowledge" and that "because the difficulty of transferring knowledge acquired from concrete instantiations may stem from extraneous information diverting attention from the relevant mathematical structure, concrete instantiations are also likely to hinder transfer for young learners who are less able than adults to control their attentional focus." However, following a replication of the original studies and analysis, [[14]] notes that “...results confirm Kaminski et al.'s findings, but the accompanying qualitative data raise serious questions about their interpretation of what students actually learned from the abstract concept exemplification. Moreover, whereas Kaminski et al. showed that abstract learners transferred what they had learned to a similar abstract context, this study shows also that students who learned from concrete examples transferred their knowledge into a similar concrete context."

The aim of our research project is not to replicate the concrete versus abstract study in the programming context, yet it is closely related: Just in the way that learning algebra beyond arithmetic is useful, there is reason to believe that enhancing traditional instruction with materials and tools to help students reason about arbitrary inputs using a symbolic approach-an analysis that requires some minimal level of abstraction and generalizationwill help students become more proficient programmers. So our goal is to study what it takes to learn the more abstract approach of reasoning with symbolic values, not using just one or the other.

The hope is that students learn to reason about code correctly, and see the benefits in other computing courses such as discrete mathematics, algorithm analysis, programming languages, and software engineering that demand analytical reasoning or writing formal assertions, contracts and invariants.

\subsection{Educational Research Questions}

The research project underlying the topics discussed in this paper considers code involving loops and recursion as well as object-based code designed with contracts, involving pre- and post-conditions. Significant data has been collected and is being analyzed. However, we focus this paper on narrower educational research questions and consider only symbolic reasoning about simple code with abstract values so that the overall presentation is meaningful. Given the growing importance of online education for the benefits to reach a diverse audience, and the role of online tools to ensure that at least a part of the CS education can happen outside a classroom, this research involves understanding the role of a reasoning tool we have built.

ERQ \#1-Learning: With or without intermediate steps, can a majority of students learn the basics of tracing code using symbolic input values, instead of specific input values (1) strictly with the help of an online reasoning tool and (2) with instruction in addition to the tool?
The hypothesis here is that a significant majority of students will indeed be able to learn to reason with symbolic values; after all, students learn algebra in high schools. But such learning will require classroom instruction in conjunction with a tool. We address the role of a step-by-step approach in reasoning correctly about code compositions and whether students will follow such an approach if learnt. We also consider if students perform as well on the reasoning questions as other topics in a CS course.

We used modified questions from the NASA TLX survey to evaluate student attitudes toward the online tool. Since the online tool was the first introduction to symbolic reasoning, it became clear (through think-alouds) that the purpose of such reasoning was not obvious to students. Since we are following a developmental evaluation ${ }^{1}$ technique (that allows new research questions to emanate), we decided to introduce the tool with a 5minute presentation explaining that the tool's purpose is to aid in reasoning about all inputs (which the better programmers can do) and raise a second question. This question concerns motivation.

Since the idea of reasoning over abstract values is a concept that is not often taught in computer science, motivation is critical. The importance of motivation is well documented in the cognitive psychology literature. In [[31]], it is noted that motivation directs behavior toward particular goals and that individuals set goals for themselves and direct their behavior accordingly. Motivation increases the amount of effort and energy that learners expend in activities directly related to their needs and goals [[13], [26], [34]]. It determines whether they pursue a task enthusiastically and wholeheartedly or apathetically and lackadaisically.

Motivation also affects what learners pay attention to and how effectively they process the information they receive [[16], [35], [37]]. For instance, motivated learners often make a concerted effort to understand classroom material-to learn it meaningfullyand consider how they might use it in their own lives. This leads to the second question.

ERQ \#2-Motivation and Attitudes: Will explaining the purpose of symbolic reasoning with an online tool impact (1) student attitudes and (2) their perception of learning?

The hypothesis based on expectancy theory here is that providing students with the tool's purpose would impact attitude towards the activity and therefore improve their motivation to complete the task successfully. Since the tool is unchanged, student learning experience should not necessarily be impacted.

We also did a demographic aggregation of data, but due to space limitations, we only make some observations without including supporting evidence.

\subsection{Summary of Research Methods}

To answer the research questions, we have experimented with a reasoning tool with and without associated instruction over three semesters in a required second-year course for CS majors on software development foundations in Java. We have varied when intermediate steps are provided in the tool activities. We have included a symbolic reasoning question on the exam to determine student learning. We have varied exam questions asking for steps, not asking for steps, and providing scaffolding. We have assessed 
student attitudes toward the reasoning tool and changes to the attitudes associated with providing a motivation for learning. ${ }^{1}$

\section{Reasoning Tool Design}

Online tutoring of programming concepts has received much attention in the CS literature (e.g., [[1], [4], [18], [23], [25], [30], [36], [42]]). There are several IDEs that provide compile-time error feedback and numerous useful capabilities (e.g., finding the declaration or all uses of a method). The novel online reasoning tool that we have developed, unlike other tutors and IDEs, is backed by the RESOLVE software verification engine [[10], [38]]. This allows it to facilitate reasoning over abstract input values. It has a strong theoretical basis and has been used for nearly a decade at multiple institutions. Thousands of undergraduate students have employed the ideas in CS courses [[12], [15], [19], [21]] and in software engineering projects [[11], [33]]. It suffices to say that the engine is far more powerful than demanded by the reasoning activities discussed here. The engine can enhance learning through a variety of logical "what if" questions. Since answers are verified automatically, answer keys are not stored or used.

\subsection{Cognitive Load Considerations}

In any learning environment, a learner can be easily frustrated by a plethora of uncontrolled possibilities of options. The goal is to tease out the role of "contextualization" in learning and prevent the cognitive capacity of a learner from being overloaded. This challenge is informed by cognitive learning theories. Specifically, cognitive load theory is concerned with the ease with which information can be processed in working memory [[32], [40]]. To solve problems, students must be able to hold multiple pieces of information in working memory at the same time. Therefore, working memory load, also known as cognitive load, is implicated as a major impediment to constructivist learning [[8]]. Interactive learning guides have the potential to decrease intrinsic and extrinsic cognitive load and help promote germane cognitive load and our tool has benefited from the excellent set of design guidelines for automated agents in [[43]] and the survey in [[2]].

\subsection{Interface Design and Reasoning Activities}

The tool has been designed with scaffolding to reduce cognitive load, as seen from the screen shot in Figure 1. The activity is at the top left with reference material below it showing only what is relevant to reason about the code on the right.

The activities begin with ones to trace code on specific input values, such as in Figure 2. The use of the tool is the first introduction to all the constructs. In the Pascal-like code shown (with which most of our students learning Java apparently have no trouble and need no introduction), we use $:=$ to denote assignments to distinguish it from the mathematical equality used in assertions. The student's task is to trace the given code and

\footnotetext{
1 Parkhurst, M., Preskill, H., Lynn, J., \& Moore, M. (2016, March 1). The Case for Developmental Evaluation. https://www.fsg.org/blog/case-developmentalevaluation
}

replace /* expression */ with the answer in the Confirm assertion (where "=" means equals, as in logic). The student can only change in the assertion. Unlike an assert statement; nothing is executed.

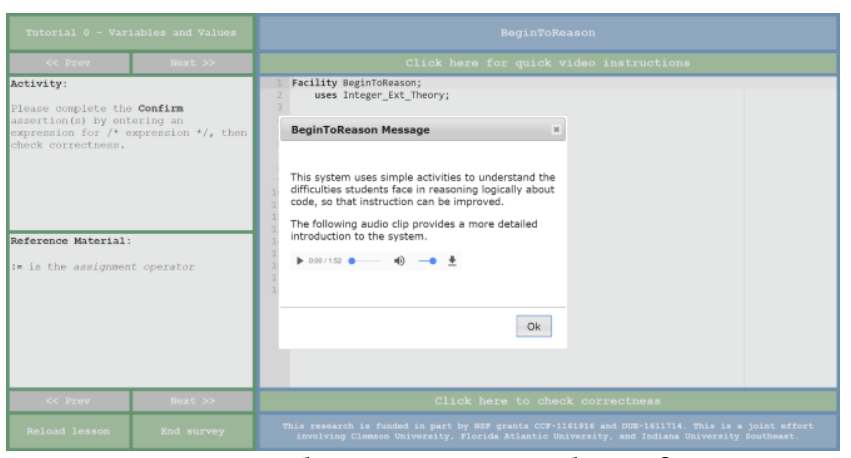

Figure 1: Online Reasoning Tool Interface

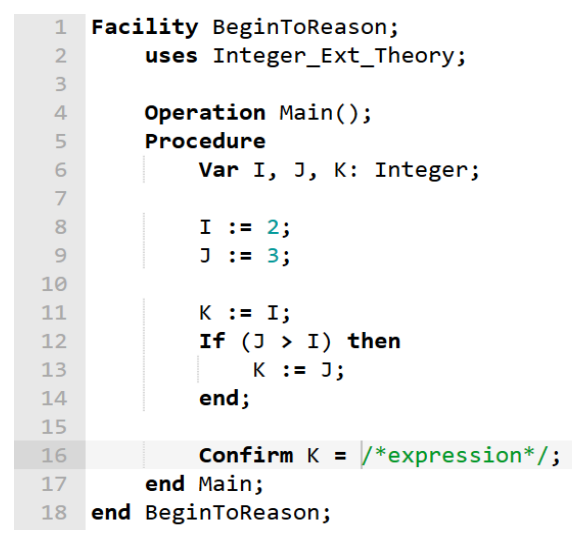

Figure 2: A Beginning Tracing Activity

An example of symbolic reasoning is shown in Figure 3.

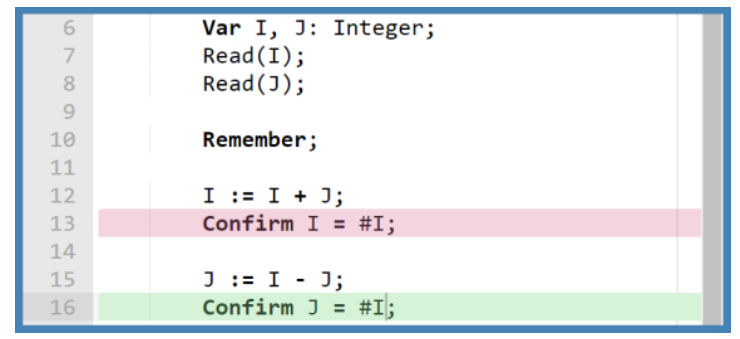

Figure 3: Example Symbolic Reasoning \& Visual Feedback

The activity asks students to state the values of $I$ and $J$ in terms of the input values, remembered to be \#I and \#J, respectively, at the line marked Remember. The tool has been designed to provide visual feedback that is immediate [[3]] and reduces cognitive load [[7], [28]]. An example of this feedback can be seen in Figure 3. Since I has changed, it is no longer true that I = \#I, and hence, line 13 is wrong (and is in red).

When the students input their answer, it could be any number of logically equivalent answers. While all answers are verified, the system expects reasonable answers, rejecting trivial answers such as Confirm I = I. When a student's answer is wrong, the 
activity may be repeated, or a follow-on activity is given. Though not a focus of the current paper, a key benefit of this tool is that it can pinpoint obstacles specific for subsets of learners [[9]].

\section{Experimental Methods}

The reasoning system and the activities were administered in closed lab sessions associated with a second year course on software development that emphasizes software engineering principles and uses Java. The course description includes specification and reasoning among the topics and is required for all CS majors at our institution. Details of this course are discussed in [[19]]. Data for the experiments discussed in this paper was obtained over the course of three semesters; fall 2016, spring 2017, and fall 2017. When there were multiple lecture sections, the students were intermingled in the lab sections.

\subsection{Teaching Using Only the Tool}

For the fall 2016 experiment, 114 students participated in the study across 5 sections of the lab. Students did not receive any formal instruction regarding symbolic reasoning before tool use. This tool served as their first introduction to symbolic reasoning about code. Though TA help was available, few students asked for any help. Students were not time constrained and took between 20 minutes to an hour to complete all the activities.

About six weeks after working with the tool in the lab, students were asked to complete a symbolic reasoning question on a regular final exam. The question was worth a total of five points, which was $20 \%$ of the exam. There were four versions of the reasoning question and the versions were randomly distributed at testing. Two versions involved reasoning about a piece of swapping code (similar, but not identical to the one given in the introduction). The other two questions had to do with the sum of two variables. These two types of questions were then further separated by one version of the question asking students to show their work, while the other did not.

\subsection{Variants and Perceptions, Without Motivation}

The spring 2017 experiment consisted of 5 lab sections with a total of 91 students. Each section was provided with one of two versions of activities embedded within the tool, which served to be their only introduction to symbolic reasoning.

Version A of the activities presented students with code to trace, and one or more Confirm assertions to complete at the bottom of the code. If students entered an incorrect answer, they were then presented with the task again, but this time provided with intermediate Confirm assertions to be established.

Version B presented students with the same code to trace, but instead gave them the intermediate Confirm assertions right away at the beginning (similar to the example seen in Figure 3). If a student entered an incorrect answer, the tool would highlight the incorrect answer and provide them with the same code and layout.

For both versions, the tool reported how many attempts each individual student made on each lesson and sub-lesson, if applicable. Students then completed a survey regarding their opinions on the tool using a Likert scale indicating how strongly they agreed or disagreed with each survey question.

Students then completed a symbolic reasoning question on their exam. The question was worth 5 points, which was $12.5 \%$ of the exam. There were four versions of the question randomly distributed. As with the previous experiment, two questions asked students to show their work, while the other two did not. These questions were then further split to where one set only used symbols $I$ and $J$ as in the lessons above, while the other incorporated a third symbol, K. After completing the exam, twenty students (diverse in demographics and grades) were invited to participate in a retrospective think-aloud protocol.

\subsection{Aided with Motivation and Instruction}

The fall 2017 experiment had a total of 92 students in 5 lab sections. All students used the activities in Version B, whereby intermediate steps were given up front. The number of attempts for each lesson was collected once again, as well as the student opinion survey. Before students interacted with the online reasoning system, the instructor gave a brief introduction explaining the purpose of the reasoning activities they were to complete during the lab. Specifically, the students were told that the best programmers were able to generalize reasoning about code on all inputs, and the goal for the research was to understand what difficulties students had in doing such reasoning, and how to develop better instructional material to communicate the ideas to all students. They were also asked to be deliberate in their effort.

Students received an hour lecture on symbolic reasoning before the exam. As part of the exam, students had to answer a symbolic reasoning question. All students received the same question, which required them to state the values of $x, y$, and $z$, after each line of code in terms of \#x, \#y, and \#z. The questions were worth 15 points, which was $15 \%$ of the exam grade.

\section{Results}

Some of our initial hypotheses held, while others did not. For some findings, the results are statistically significant, and in others, they are not. We have also collected information on student answers during the online activities. For example, it is easy to mine for common wrong answers and provide guided tutoring. We have done screen captures of student work from fall 2017 to supplement other collected information. Though we do not include a detailed analysis of the think-alouds in this paper, we note that they have guided one of our design decisions.

\subsection{ERQ \#1: Ability to Learn Symbolic Reasoning}

Across all versions of the questions in fall 2016, students received an average of at least 4 points, which translates into completing approximately $80 \%$ of the question correctly. It would appear the online tool can successfully introduce all students to the basics of symbolic reasoning. At the same time, it is equally possible that the question was too easy. This is a useful, but not 
definitive result. Subsequent exam questions were more demanding (in terms of the number of variables and statements).

\section{Role of Intermediate Steps}

Figures $6 \mathrm{a}$ and $6 \mathrm{~b}$ are based on spring 2017 data, where half the students used Version A activities, where intermediate steps were given only to students who were having difficulties, and half the students were given Version B activities, with intermediate steps from the beginning. It would appear that Version A of the activities were more successful at assisting students with symbolic reasoning. $37 \%$ of students were able to successfully trace the code for Version A on the first try, while only $24 \%$ were able to do so on Version B. Are intermediate steps not helpful?

There are several confounding factors. There were more assertions to complete in Version B, because of intermediate steps. There were four for one activity in Version B compared to two for version $\mathrm{A}$, so fewer students got through the activity in one try.

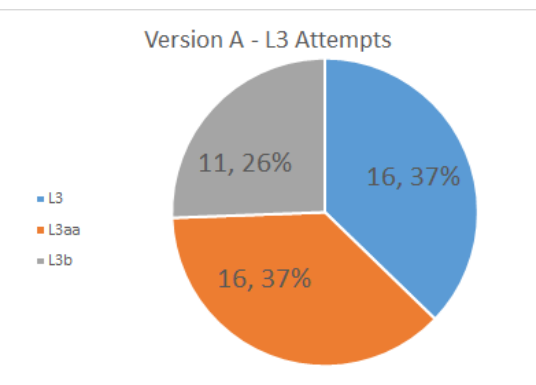

Figure 6a: Activity Version A with Steps Given As Needed

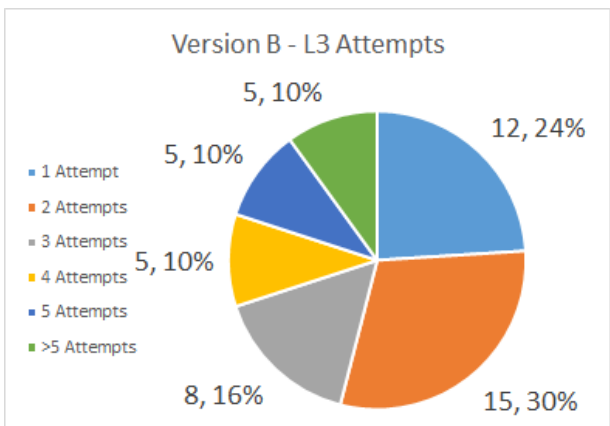

Figure 6b: Activity Version B with Steps Given Initially

In addition, in Version A, when a student provided an incorrect response, they were provided with a version with intermediate steps (similar to B), and when students failed that, it shortened the problem (L3b in the figure). By the time a student reached this lesson, she had seen a version of this problem 4 times. A detailed discussion of this particular analysis to pinpoint specific student learning obstacles is the topic of [[12]].

Another source of complication was the inability to account for submissions where student work had not been completed. Version B may have required 4 Confirm statements, and if one were left blank when the student submitted to check progress, it would be considered an incorrect attempt to solve the problem.

In the think-alouds, we noticed that a subset of students who made errors on Activity 3 did seem to understand the reasoning process, but made algebraic errors only because they insisted on doing it "in their heads." While we offered a sheet of paper to help with the process, only a small fraction of the students took up the offer. For this group, a step-by-step process would have been exactly what would be helpful. Though the sample size is limited, we also noticed that female and African-American students did as well as others with Version B. These observations along with observations on exam answers convinced us to switch to version B activities with intermediate steps for all subsequent sections.

\section{Learning Reasoning w/wo Classroom Instruction}

The fall 2016 course was taught in a single large lecture section (which was not repeated). To keep the comparison meaningful, we compare only spring 2017 and fall 2017 data. We used a MannWhitney U-test because it is more suitable to compare performance of different groups of students across semesters and because it does not require that the two independent samples are normally distributed.

In fall 2017, tool-based instruction was supplemented with a one-hour classroom lecture. The difference in scores for the exam reasoning question between the semesters as seen in Figure 7(a) was found to be statistically significant with a $\mathrm{p}$ value less than 0.05 . In the spring, only $59.1 \%$ of students completed at least $80 \%$ of the question correctly, while in the fall, $74.2 \%$ did. A similar pattern is observed between the overall exam scores, where fall students performed significantly better, with a $\mathrm{p}$ value of 0.0005 . While this would point directly to the benefit of classroom instruction, the topic of motivation detailed in the next subsection is a confounding factor. Regardless the important point is that symbolic reasoning can be learned by a majority of students.

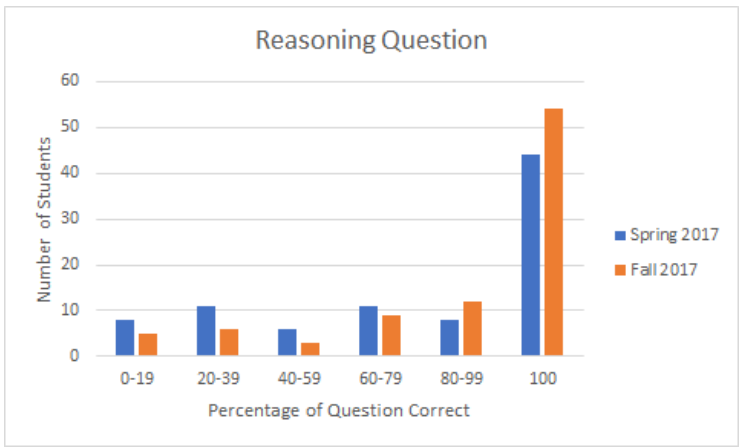

Figure 7a: Performance, w/wo Supplemental Instruction

\subsection{ERQ \#2: Motivation and Attitudes}

To evaluate students' attitudes towards the tool, we used questions from the NASA TLX survey [[29]], with minor variations to the questions, and a major variation in using a 5point Likert scale, instead of a continuous scale (due to limitations of our course management system.) There were two background 
questions and our analysis showed there was no statistically significant difference between the two semesters.

Q1 My prior preparation in CS is good

Q2 My performance in the current course is good

There were ten other questions. Agree or strongly agree are the desired outcomes for the first eight.

Q3 The interface was easy to use

Q4 I was able to understand what I needed to do

Q5 I found this to be a useful learning experience

Q6 The feedback from the system was helpful

Q7 I found the activities engaging

Q8 The task was mentally demanding

Q9 I was successful in accomplishing what I was asked to do

Q10 I had to work hard to accomplish my level of performance

Disagree or strongly disagree are the desired outcomes for the last two:

Q11 The pace of the task was hurried or rushed

Q12 I was insecure, discouraged, irritated, stressed, and annoyed

Each answer was assigned a numerical value according to the desired outcome. For example whereas for the question "The interface was easy to use" a response of Strongly Agree is coded as a 5, for the question "The pace of the task was hurried or rushed" Strongly Disagree is coded as a 5.

When comparing the subset of students who used version B from the spring of 2017 with the students from the fall of 2017 (who also used an identical version B), there is an observable difference in student attitudes regarding use of the online tool. This is seen in Figure 8.

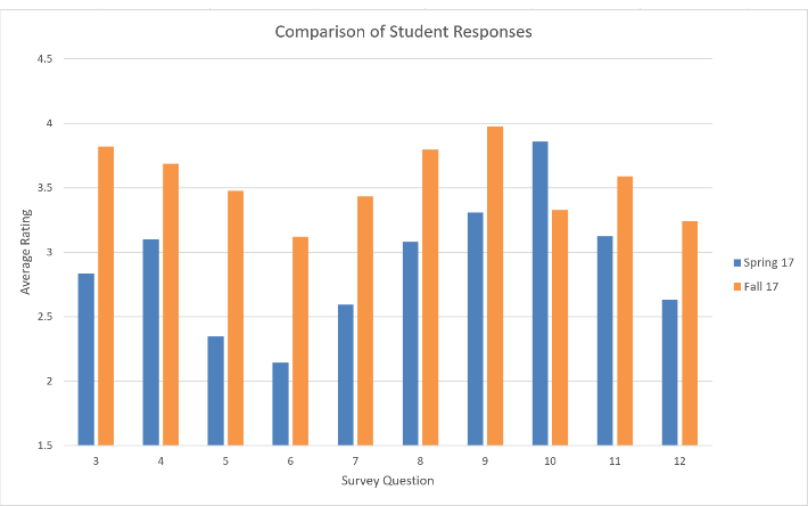

Figure 8: Impact of Motivation on Attitudes, Q3 to Q12

For questions 3 through 12, excluding question 8 , student answers were more favorable in the range of 0.47 to 1.12 . The response for question 8 is also favorable because it shows that students felt like they did not have to work as hard to achieve the same level of success. A Mann-Whitney U-test indicated that questions 3 through 12 had a statistically significant difference in answers when using an alpha value of 0.05 .
This evidence indicates that student attitudes towards the reasoning tool and use of the tool are altered when presented with a brief introduction that provides students with a purpose and motivation to complete the task. Important to note is the significance of the response to the agreement of the statements "I found this to be a useful learning experience," and "The feedback from the system was helpful," and the disagreement to the statement "I was insecure, discouraged, irritated, stressed, and annoyed"-even though the activities were identical across groups. So our hypothesis to part (1) of ERQ \#2 was correct, but part (2) about their perception of learning from the tool was wrong. Motivation was important on both counts. The positive results from motivation held across all demographics.

\section{Discussion and Conclusions}

Our research has shown it is possible to teach symbolic reasoning to a majority of students (at the $80 \%$ or a B-grade level). Classroom instruction and providing a motivational purpose for the tool have a statistically significant impact. Intermediate steps have been found to be useful especially for underrepresented students.

One potential threat to validity of the results is that each semester had slight variations in the experimental conditions. Using Mann-Whitney U-test on the incoming student GPA, we found that the spring and fall student populations were comparable. While both semesters were taught by the same instructor, spring was the first time this instructor taught this course. This could indicate that the instructor was better equipped to teach these concepts, and why students performed well on this particular exam. Upon further examination, overall average course grade for the fall semester is actually worse than the spring semester indicating that students did not receive an advantage due to teacher experience.

We have conducted extensive think-alouds to learn more about student thinking though we do not report the results here. Without prompting, some students explained to us how they might use symbolic reasoning in other programming and algorithms courses, suggesting that benefits might be more far reaching. Some others had difficulty with the basics of algebra, so exploring deficiencies in prior learning is a possible research direction.

\section{ACKNOWLEDGMENTS}

We acknowledge the contributions of several members of our research groups at Clemson, Florida Atlantic University, and Ohio State University. Special thanks are due to Bruce Weide. This work is funded in part by US National Science Foundation grants CCF1161916, DUE-1611714, DUE-1609718, and DUE-1610957.

\section{REFERENCES}

[1] V. Aleven, B. M. McLaren, and J. Sewall. 2009. Scaling Up Programming by Demonstration for Intelligent Tutoring Systems Development: An OpenAccess Web Site for Middle School Mathematics Learning. IEEE Trans. on Learning Technologies 2, 64-78. 
[2] J. R. Anderson, A. T. Corbett, K. R. Koedinger, and R. Pelletier. 1995. Cognitive Tutors: Lessons Learned, The Journal of the Learning Sciences 4 , 167-207.

[3] R. Azevedo and R. M. Bernard. 1995. A Meta-Analysis of the Effects of Feedback in Computer-Based Instruction. Journal of Educational Computing Research 13, 2, $111-127$.

[4] P. Bhattacharya, L. Tao, B. W. Kai Qian, and E. K. Palmer. 2011. A Cloudbased Cyberlearning Environment for Introductory Computing Programming Education. In Procs. 11th ICALT, 12-13.

[5] P. Bucci, T. Long, and B. Weidi. 2001. Do We Really Teach Abstraction?, In Proceedings of the 32nd SIGCSE Technical Symposium on Computer Science Education, ACM, 26-30.

[6] K. J. Carbonneau, S. C. Marley, J. P. Selig. 2013. A meta-analysis of the efficacy of teaching mathematics with concrete manipulatives, Journal of Educational Psychology 105, No. 2, 380-400. http://dx.doi.org/10.1037/a0031084

[7] C. Chen, S. Pedersen, and K. L. Murphy. 2011. Learners' perceived information overload in online learning via computer-mediated communication. Research in Learning Technology, 19, 1, 101-116.

[8] M. P. Cook, "Visual representations in science education: The influence of prior knowledge and cognitive load theory on instructional design principles," Science Education 90, 6, 2006, 1073-1091.

[9] M. P. Cook, M. Fowler, J. O. Hallstrom, J. E. Hollingsworth, T. Schwab, Y. S. Sun, \& M. Sitaraman, 2018. Where Exactly are the Difficulties in Reasoning Logically about Code? Experimentation with an Online System. In Proceedings of the 23rd Annual ACM Conference on Innovation and Technology in Computer Science Education (pp. 39-44). ACM.

[10] C. T. Cook, H. Harton, H., Smith, and M. Sitaraman, 2012. Specification Engineering and Modular Verification Using a Web-Integrated Verifying Compiler, In 2012 34th International Conference on Software Engineering (ICSE), 2012, 1379-1382.

[11] C. T. Cook, S. Drachova-Strang, Y-S. Sun, M. Sitaraman, J. C. Carver, and J. E. Hollingsworth, 2013. Specification and Reasoning in SE Projects Using a Web IDE, In Proceedings Conference on Software Engineering Education \& Training (CSSE\&T), 2013

[12] Cook, C. T., Drachova, S., Hallstrom, J. O., Hollingsworth, J. E., Jacobs, D. P., Krone, J., \& Sitaraman, M. 2012. A Systematic Approach to Teaching Abstraction and Mathematical Modeling, In Proceedings of the 17th ACM Annual Conference on Innovation and Technology in Computer Science Education (ITiCSE '12), ACM, New York, NY, USA, 357-362.

[13] M. Csikszentmihalyi, and J. Nakamura. 1989. The dynamics of intrinsic motivation: A study of adolescents. In R. Ames \& C. Ames (Eds.), Research on motivation in education: Goals and cognitions (pp. 45-71). New York: Academic Press.

[14] D. De Bock, J. Deprej, W. van Duren, M. Roelens, and L. Verschaffel. 2011 Abstract or Concrete Examples in Learning Mathematics? A Replication an Elaboration of Kaminsky, Stoutsky, and Heckler's Study. Journal for Research in Mathematics Education 42, 2, 109-126.

[15] S. Drachova-Strang, J. O. Hallstrom, J. E. Hollingsworth, J. Krone, R. Pak, and M. Sitaraman, "Teaching Mathematical Reasoning Principles for Software Correctness and Its Assessment," ACM Transactions on Computing Education 15, 3, Article 15 (August 2015), 22 pages.

[16] J. S. Eccles, and A. Wigfield. 2002. Motivational beliefs, values, and goals. Annual Review of Psychology, 53, 109-132.

[17] S. Fitzgerald, B. Simon, and L. Thomas. 2005. Strategies that students use to trace code: an analysis based in grounded theory. In Procs. ICER '05 ACM, NY, USA, 69-80.

[18] P. Guo. 2018. http://www.pythontutor.com/java.html\#mode=edit

[19] J. O. Hallstrom, C. Hochrine, J. Sorber, \& M. Sitaraman, 2014. An ACM 2013 exemplar course integrating fundamentals, languages, and software engineering. In Proceedings of the 45th ACM technical symposium on Computer science education (pp. 211-216). SIGCSE '14, ACM, New York, NY, USA.

[20] P. B. Henderson. 2003. Mathematical Reasoning in Software Engineering Education. Communications of the ACM 46, 9, 45-50.

[21] W. Heym, P. A. Sivilotti, P. Bucci, M. Sitaraman, K. Plis, J. E. Hollingsworth, \& N. Sridhar, (2017, November). Integrating Components, Contracts, and
Reasoning in CS Curricula with RESOLVE: Experiences at Multiple Institutions. In Software Engineering Education and Training (CSEE\&T), 2017 IEEE 30th Conference on (pp. 202-211). IEEE.. 2017. CSEE\&T, IEEE, NY, USA.

[22] J. A. Kaminsky, V. M. Stoutsky, A. F. Heckler. 2008. The Advantage of Abstract Examples in Learning Math, Learning Theory, Science 320, 454-455.

[23] R. Kumar, C. P. Rose, Y. Wang, M. Joshi, and A. Robinson. 2007. Tutorial Dialog as Adaptive Collaborative Learning Support. In Procs. Conference on Artificial Intelligence in Education: Building Technology Rich Contexts That Work, IOS Press, 383-390.

[24] C. M. Lewis. 2014. Exploring variation in students' correct traces of linear recursion. In Procs. ICER '14. ACM, NY, USA, 67-74.

[25] C. Li, Z. Dong, R. Untch, Chasteen, and N. Reale. 2011. PeerSpace-An Online Collaborative Learning Environment for Computer Science Students. In Procs. 11th ICALT, 409-411.

[26] M. L. Maehr. 1984. Meaning and Motivation: Toward a Theory of Personal Investment. Research on Motivation in Education, 1, 115-144.

[27] W. McCallum. 2008. Commentary on Kaminsky, et al, The Advantages of Abstract Examples in Learning Math, Science.

[28] R. Moreno. 2004. Decreasing Cognitive Load for Novice Students: Effects of Explanatory versus Corrective Feedback in Discovery-Based Multimedia. Instructional Science 32, 99-113.

[29] NASA, 1986. NASA Task Load Index (TLX) v. 1.0 Manual.

[30] C. O'Brien, M. Goldman, and R. C. Miller. 2014. Java tutor: bootstrapping with python to learn Java. Proceedings of the first ACM conference on Learning. ACM, New York, NY, USA. 185-186. 10.1145/2556325.2567873.

[31] J. E. Ormond. 2014. Educational Psychology: Developing Learners. Upper Saddle River, NJ: Pearson.

[32] F. Paas, T. van Gog, and J. Sweller. 2010. Cognitive load theory: New conceptualizations, specifications, and integrated research perspectives. Educational Psychology Review, 22, 2, 115-121.

[33] C. Priester, Y-S. Sun, and M. Sitaraman, "Tool-Assisted Loop Invariant Development and Analysis", In Proceedings of the $29^{\text {th }}$ Conference on Software Engineering Education and Training (CSEE\&T 2016), IEEE, NY, USA, 66-70

[34] P. R. Pintrich, D. Smith, T. Garcia, and W. McKeachie. 1993. Predictive validity and reliability of the Motivated Strategies for Learning Questionnaire (MSLQ). Educational and Psychological Measurement, 5, 801-813.

[35] P. R. Pintrich, and D. H. Schunk. 2002. Motivation in education: Theory, research, and applications (2nd ed.). Upper Saddle River, NJ: Prentice Hall.

[36] T. W. Price, Y. Dong, and D. Lipovac. 2017. iSnap: Towards Intelligent Tutoring in Novice Programming Environments. In Procs. SIGCSE '17, ACM, NY, USA, 483-488.

[37] K. J. Pugh, and D. A. Bergin. 2006. Motivational influences on transfer. Educational Psychologist, 41, 147 - 160.

[38] M. Sitaraman, B. Adcock, J. Avigad, D. Bronish, P. Bucci, D. Frazier, H. M. Friedman, H. Harton, W., Heym, J., Kirschenbaum, J., Krone, H. Smith, and B. W. Weide., "Building a Push button RESOLVE Verifier: Progress and Challenges," In Formal Aspects of Computing, Springer, 2011, 607-626.

[39] J. C. Spohrer, and E. Soloway. 1986. Novice mistakes: are the folk wisdoms correct? Communications of the ACM 29, 7, 624-632.

[40] J. Sweller, P. Ayres, and S. Kalyuga, 2011. Cognitive load theory. Springer, NY.

[41] Web-CAT is an advanced automated grading system that can grade students on how well they test their own code. http://web-cat.org/home

[42] J. B. Wiggins, et al. 2015. JavaTutor: An Intelligent Tutoring System that Adapts to Cognitive and Affective States during Computer Programming. In Procs. SIGCSE '15, ACM, NY, USA, 599-599.

[43] P. Wouters, F. Paas, and J. J. G. van Merriënboer. 2008. How to Optimize Learning from Animated Models: A Review of Guidelines Based on Cognitive Load. Review of Educational Research.

[44] B. Xie, G. L. Nelson, and A. J. Ko. 2018. An Explicit Strategy to Scaffold Novice Program Tracing. In Proceedings of the 49th ACM Technical Symposium on Computer Science Education (SIGCSE '18). ACM, New York, NY, USA, 344-349. DOI: https://doi.org/10.1145/3159450.3159527 